\title{
Research on the Risk Factors of Material Misstatement of Listed Companies in China
}

\author{
Wang Lan ${ }^{1, a}$, Zhang $\mathrm{He}^{2, b^{*}}$ \\ ${ }^{1}$ College of management, Xi'an University of Science and Technology, Xi'an, Shaanxi, China \\ ${ }^{2}$ College of management, Xi'an University of Science and Technology, Xi'an, Shaanxi, China \\ a958932865@qq.com \\ b*517318916@qq.com
}

\begin{abstract}
Since the 21st century, with the rapid development of the global economy, economic business is complex and diverse. At the same time, financial fraud and audit failure cases occur frequently at home and abroad. Under the modern risk oriented audit mode, the identification, evaluation and response of the risk of material misstatement become the key to reduce the audit risk. This paper selects nine factors that are expected to have a significant impact on the risk of material misstatement of Listed Companies in China as the independent variables of the empirical study, takes the risk level of material misstatement of Listed Companies in China as the dependent variable of the empirical study, and selects 733 listed companies with non-standard audit opinions from 2016 to 2019 as the research samples to describe the research data The results of empirical research are as follows: statistical analysis, correlation analysis and multiple regression analysis.
\end{abstract}

Keywords: Audit; listed companies, risk of material misstatement, influencing factors, empirical research

\section{我国上市公司重大错报风险影响因素研究 \\ 王岗 $1, \mathrm{a}$ 张赫 $2, \mathrm{~b}^{*}$}

1 西安科技大学管理学院, 西安, 陕西, 中国

2 西安科技大学管理学院, 西安, 陕西, 中国

a958932865@qq.com

b*517318916@qq.com

\section{摘要}

21 世纪以来，全球经济迅猛发展，经济业务复杂多样，同时国内外财务造假和审计失败案例也频繁发生。在 现代风险导向审计模式下，重大错报风险的识别、评估和应对成为了降低审计风险的关键。本文选取了 9 个预 期会对我国上市公司的重大错报风险产生显著作用的影响因素作为实证研究的自变量, 以我国上市公司的重 大错报风险水平作为实证研究的因变量，同时选择 2016-2019 年我国被出具非标准审计意见的 733 家上市公 司作为研究样本, 对研究数据进行了描述性统计分析、相关性分析和多元回归分析, 得到了较好的实证研究结 果。

关键词: 审计; 上市公司; 重大错报风险; 影响因素; 实证研究

\section{1 引言}

从 21 世纪初发生的安然事件、蓝田事件和银广 厦事件等大量审计失败的典型案例到近几年发生的 康美药业、康得新和瑞幸咖啡等知名企业的巨额财务 造假事件, 这些触目惊心的警示案例对整个经济和审
计环境造成了巨大的冲击。为了进一步发挥审计在合 理保证财务信息有效性中的作用，同时弥补传统审计 方法的缺陷, 国际审计与鉴证准则理事会和我国财政 部分别于 2003 年和 2006 年在审计准则中引入了现代 风险导向审计方法, 重大错报风险这一概念由此引入。 《中国注册会计师审计准则第 1211 号——通过了解 
被审计单位及其环境识别和评估重大错报风险》对重 大错报风险的定义为: 重大错报风险是指财务报表在 审计前存在重大错报的可能性 ${ }^{[1]}$ 。

\section{2 我国上市公司重大错报风险现状概述}

2016-2019 年我国上市公司的数量分别为 3136、 3513、3607、3814 家, 呈现出逐年递增的趋势; 20162019 年我国上市公司财务报告被出具非标准审计意 见的数量分别为 $107 、 130 、 223 、 273$ 家，同样呈现出 逐年递增的趋势；2016-2019 年我国被出具非标准审 计意见的上市公司所占比例分别为 $3.41 \% 、 3.70 \%$ 、 $6.18 \% 、 7.16 \%$, 依旧呈现出逐年递增的趋势。以上数 据说明我国被出具非标准审计意见上市公司数量的 增长速度高于我国上市公司总体数量的增长速度, 反 映出近四年来我国上市公司存在的问题逐渐增多, 重 大错报风险逐渐升高, 审计情况不容乐观。除“否定意 见”审计意见近四年只出现了 1 次外, 其他三种非标 准审计意见的数量基本上呈现出逐年递增的趋势。其 中, “无保留意见加事项段”审计意见的数量占比最大, 其次是“保留意见”审计意见, 再次是“无法表示意见” 审计意见，最后是“否定意见”审计意见。

\section{3 我国上市公司重大错报风险影响因素的实证 研究设计}

\section{1 我国上市公司重大错报风险影响因素假设}

本文在综合了众多国内外学者之前的有关研究 的基础之上, 参考了我国上市公司的主要特点, 根据 我国上市公司重大错报风险的现状, 推出以下影响因 素, 并根据提出的影响因素建立如下假设以待后文的 进一步研究。

H1: 企业出现巨额或连续亏损, 持续经营能力下 降, 对重大错报风险水平有显著影响; $\mathrm{H} 2$ : 企业流动 负债较多或存在大额借款未偿还现象, 对重大错报风 险水平有显著影响; H3: 企业发生重大收购、重组或 其他非经常性事项，对重大错报风险水平有显著影响; $\mathrm{H} 4$ : 企业存在重大的投资、融资或股权转让项目，对 重大错报风险水平有显著影响; H5: 企业存在重大的 关联方交易或资金占用情况，对重大错报风险水平有 显著影响; H6: 企业的内部控制存在重大缺陷或失效, 对重大错报风险水平有显著影响; H7: 企业以往存在 重大错报风险或本期期末出现重大会计调整, 对重大 错报风险水平有显著影响; H8: 企业的经营活动或财 务报告受到监管机构的调查或处罚, 对重大错报风险 水平有显著影响; H9: 企业存在未决诉讼、仲裁或案 件，对重大错报风险水平有显著影响。

\section{2 样本选取}

本文选取 2016-2019 年我国被出具非标准审计意 见的 733 家上市公司作为实证研究样本, 其中 20162019 年的上市公司样本数量分别为 107、130、223、
273 家。

\section{3 变量设计}

在研究中, 本文以我国上市公司重大错报风险水 平作为被解释变量, 将九个影响因素作为解释变量, 两个影响因素作为控制变量。具体变量设计如下:

\subsection{1 被解释变量设计}

本文选用我国上市公司重大错报风险水平 (RMM) 作为被解释变量。根据《中国注册会计师审 计准则第 1501 号——审计报告》与《中国注册会计 师审计准则第 1502 号——非标准审计报告》的准则 精神, 同时参考张宜霞和郭玉 (2015) 的相关研究方

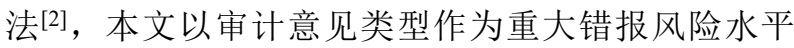

(RMM) 的替代变量, 将被出具不同类型审计意见的 我国上市公司的重大错报风险水平 (RMM) 分为五类。 其中, 被出具标准无保留意见的上市公司的重大错报 风险水平 $(R M M)$ 为 0 ; 被出具带强调事项段的无保 留意见的上市公司的重大错报风险水平 (RMM) 为 0.25 ; 被出具保留意见的上市公司的重大错报风险水 平 (RMM) 为 0.5 ; 被出具无法表示意见的上市公司 的重大错报风险水平 $(R M M)$ 为 0.75 ; 被出具否定意 见的上市公司的重大错报风险水平 (RMM) 为 1 。

\subsection{2 解释变量设计}

根据研究假设中的九个影响因素, 并参考现有的 研究成果, 本文设置了相应的解释变量: 公司出现巨 额或连续亏损（H1），根据企业年报利润表中的营业 利润数据确定企业的年度经营状况与亏损数额, 将企 业的年度亏损数额与总资产的比值设置为 $\mathrm{H} 1$, 若企 业当年未出现巨额或连续亏损，则用 0 表示。公司存 在流动负债较多或大额借款未偿还现象（H2）, 根据 企业年报资产负债表中的流动负债数据确定企业的 年度负债与借款情况, 将企业的年度流动负债数额与 总资产的比值设置为 $\mathrm{H} 2$, 若企业当年不存在流动负 债较多或大额借款未偿还现象, 则用 0 表示。公司发 生重大收购、重组或其他非经常性事项（H3）, 若企 业当年发生了重大收购、重组或其他非经常性事项, 则用 1 表示，否则用 0 表示。公司存在重大的投资、 融资或股权转让项目 (H4), 若企业当年存在重大的 投资、融资或股权转让项目，则用 1 表示，否则用 0 表示。公司存在重大的关联方交易或资金占用情况

（H5），若企业当年存在重大的关联方交易或资金占 用情况，则用 1 表示，否则用 0 表示。公司内部控制 存在重大缺陷或失效（H6），若企业当年的内部控制 存在重大缺陷或失效, 则用 1 表示, 否则用 0 表示。 公司以往存在重大错报风险或本期期末出现重大会 计调整 (H7), 若企业在以往的年报审计中被出具过 非标准审计意见或在当期期末出现了重大的会计调 整，则用 1 表示，否则用 0 表示。公司经营活动或财 务报告受到监管机构的调查或处罚 (H8), 若企业当 
年的经营活动或财务报告受到了监管机构的调查或 处罚，则用 1 表示，否则用 0 表示。公司存在未决诉 讼、仲裁或案件 (H9)，若企业当年存在未决诉讼、 仲裁或案件，则用 1 表示，否则用 0 表示。

\subsection{3 控制变量设计}

本文选取企业规模（SIZE）和上市地点（PLA） 作为两个控制变量: 通过文献研究发现学者 Dhaliwal 等人 (2011) 将企业规模作为实证研究中的控制变量 [3]，同时学者王建玲等人（2016）也将企业规模以企 业总资产的自然对数形式作为实证研究中的控制变 量 ${ }^{[4]}$ 。企业的总资产可以从年度财务报告中获取真实 可靠的数据, 增加了研究的可靠性。因此, 本文选用 企业总资产的自然对数作为企业规模 (SIZE) 的衡量 标准, 以其作为本文实证研究的控制变量。通过文献 研究发现学者宋海风 (2014) 将企业的上市地点作为 实证研究中的控制变量 ${ }^{[5]}$ 。由于不同上市地点的不同 上市类型的上市公司所面对的证券交易所对其提出 的相关要求和规定不同, 因此本文对不同上市地点的 不同上市类型的上市公司进行分类, 将深市 A 股上市 公司设置为 0 , 将沪市 $\mathrm{A}$ 股上市公司设置为 1 , 将创 业板上市公司设置为 2 , 将其他类型上市公司设置为 3 , 以此作为本文实证研究的控制变量。

\section{4 模型构建}

根据本文研究的 9 个影响因素对我国上市公司重 大错报风险水平 (RMM) 的影响, 根据上文的理论研 究以及假设, 建立以下多元回归模型:

$$
\begin{aligned}
& \mathrm{RMM}=\alpha+\beta_{1} \mathrm{H} 1+\beta_{2} \mathrm{H} 2+\beta_{3} \mathrm{H} 3+\beta_{4} \mathrm{H} 4+\beta_{5} \mathrm{H} 5+\beta_{6} \mathrm{H} 6 \\
& +\beta_{7} \mathrm{H} 7+\beta_{8} \mathrm{H} 8+\beta_{9} \mathrm{H} 9+\beta_{10} \mathrm{SIZE}+\beta_{11} \mathrm{PLA}+\varepsilon \\
& \text { 其中, } \alpha \text { 为常数项, } \beta_{1} 、 \beta_{2} 、 \beta_{3} 、 \beta_{4} 、 \beta_{5} 、 \beta_{6} 、 \beta_{7} 、
\end{aligned}
$$
$\beta_{8} 、 \beta_{9} 、 \beta_{10} 、 \beta_{11}$ 分别为解释变量系数, $\varepsilon$ 为误差项。

\section{4 实证研究与结果分析}

文章使用 IBM SPSS Statistics 21 对研究数据进行 了描述性统计分析、相关性分析和多元回归分析, 得 出相关研究结果及分析如下:

\section{1 描述性统计分析}

表 1 描述性统计结果

\begin{tabular}{cccccc}
\hline & $\mathrm{N}$ & 极小值 & 极大值 & 均值 & 标准差 \\
\hline $\mathrm{RMM}$ & 733 & .25 & 1.00 & .4229 & .18488 \\
$\mathrm{H} 1$ & 733 & .00 & 25.68 & .2023 & 1.03884 \\
$\mathrm{H} 2$ & 733 & .00 & 23.44 & .3406 & 1.04534 \\
$\mathrm{H} 3$ & 733 & .00 & 1.00 & .1446 & .35195 \\
$\mathrm{H} 4$ & 733 & .00 & 1.00 & .3765 & .48485 \\
$\mathrm{H} 5$ & 733 & .00 & 1.00 & .1801 & .38452 \\
$\mathrm{H} 6$ & 733 & .00 & 1.00 & .0941 & .29221 \\
\hline
\end{tabular}

\begin{tabular}{cccccc}
\hline $\mathrm{H} 7$ & 733 & .00 & 1.00 & .7937 & .32899 \\
$\mathrm{H} 8$ & 733 & .00 & 1.00 & .2456 & .43072 \\
$\mathrm{H} 9$ & 733 & .00 & 1.00 & .3151 & .46489 \\
$\mathrm{SIZE}$ & 733 & 16.65 & 26.14 & 21.7229 & 1.43534 \\
$\mathrm{PLA}$ & 733 & .00 & 3.00 & .6467 & .73689 \\
有效的 N & 733 & & & & \\
\hline
\end{tabular}

由表 1 我们可以看出, RMM 的均值为 0.42 , 说 明这 733 家上市公司的重大错报风险平均水平为 0.42 , 处于一个中等水平。同时我们从表格中还可以看出, RMM 的极大值为 1 , 极小值为 0.25 , 两者差距为 0.75 , 说明这些样本公司之间的重大错报风险水平是存在 较大的个体差异的。

自变量指标方面, 我们可以看到: $\mathrm{H} 1$ 的均值为 0.20 , 说明样本公司的年度亏损数额与总资产之比的 平均值为 $20 \%$; 同时, $\mathrm{H} 1$ 的极大值与极小值分别为 25.68 和 0, 说明样本公司的经营状况存在较大差异, 出现巨额亏损与并未亏损的样本公司同时存在。 $\mathrm{H} 2$ 的均值为 0.34 , 说明样本公司的年度流动负债数额与 总资产之比的平均值为 $34 \%$; 同时, $\mathrm{H} 2$ 的极大值与 极小值分别为 23.44 和 0 , 说明样本公司的负债与借 款情况存在较大差异, 出现巨额流动负债与并未出现 大额流动负债与借款的样本公司同时存在。其他指标 方面， H3、H4、H5、H6、H7、H8、H9 的均值分别 为 $0.14 、 0.38 、 0.18 、 0.09 、 0.79 、 0.25 、 0.32$ 。

控制变量指标方面, SIZE 的均值为 21.72 , 极大 值与极小值分别为 26.14 和 16.65 , 两端的值之间具有 一定的差距, 说明在样本公司里面, 既有规模巨大的 巨无霸企业, 也有经济体量较小的小型企业。PLA 的 均值为 0.65 , 说明样本公司的上市地点与上市类型在 数量分布上较为均匀, 有利于后续研究的进行。

\begin{tabular}{|c|c|c|c|c|c|c|c|}
\hline & & RMM & $\mathrm{H} 1$ & $\mathrm{H} 2$ & $\mathrm{H} 3$ & $\mathrm{H} 4$ & $\mathrm{H} 5$ \\
\hline & Pearson 相 & 1 & $.092^{*}$ & $.082^{*}$ & $.156^{* *}$ & $.317^{* *}$ & $.397^{* *}$ \\
\hline $\mathrm{R}$ & 关性 & & & & & & \\
\hline M & 显著性 & & .013 & .026 & .000 & .000 & .000 \\
\hline \multirow[t]{3}{*}{ M } & （双侧） & & & & & & \\
\hline & $\mathrm{N}$ & 733 & 733 & 733 & 733 & 733 & 733 \\
\hline & & H6 & $\mathrm{H} 7$ & $\mathrm{H} 8$ & $\mathrm{H} 9$ & SIZE & PLA \\
\hline & Pearson 相 & $.400^{* *}$ & $.076^{*}$ & $.221^{* *}$ & $.343^{* *}$ & $.163^{* *}$ & .000 \\
\hline $\mathrm{R}$ & 关性 & & & & & & \\
\hline M & 显著性 & .000 & .039 & .000 & .000 & .000 & .992 \\
\hline \multirow[t]{2}{*}{ M } & （双侧） & & & & & & \\
\hline & $\mathrm{N}$ & 733 & 733 & 733 & 733 & 733 & 733 \\
\hline
\end{tabular}

\section{2 相关性分析}

表 2 Pearson 相关系数表 
由表 2 我们可以看出, 因变量 $\mathrm{RMM}$ 和自变量 $\mathrm{H} 3 、 \mathrm{H} 4 、 \mathrm{H} 5 、 \mathrm{H} 6 、 \mathrm{H} 8 、 \mathrm{H} 9$ 及控制变量 SIZE 在 0.01 水平（双侧）上显著正相关; 因变量 RMM 和自变量 $\mathrm{H} 1 、 \mathrm{H} 2 、 \mathrm{H} 7$ 在 0.05 水平（双侧）上显著正相关; 因 变量 RMM 和控制变量 PLA 不存在相关性。

Pearson 相关系数方面, 我们可以看到: 因变量 RMM 和自变量 H6 的 Pearson 相关系数为 0.400 , 说 明两者之间的相关强度为中等程度相关; 因变量 RMM 和自变量 H4、H5、H8、H9 的 Pearson 相关系 数分别为 $0.317 、 0.397 、 0.221 、 0.343$, 介于 $0.2-0.4$ 之 间, 说明因变量 RMM 和这些自变量之间的相关强度 为弱相关; 因变量 RMM 和自变量 H1、H2、H3、H7 及控制变量 SIZE 的 Pearson 相关系数分别为 0.092 、 $0.082 、 0.156 、 0.076 、 0.163$, 低于 0.2 , 说明因变量 RMM 和这些自变量及控制变量之间的相关强度更弱。

\section{3 多元回归分析}

表 3 线性回归与多重共线性检验结果

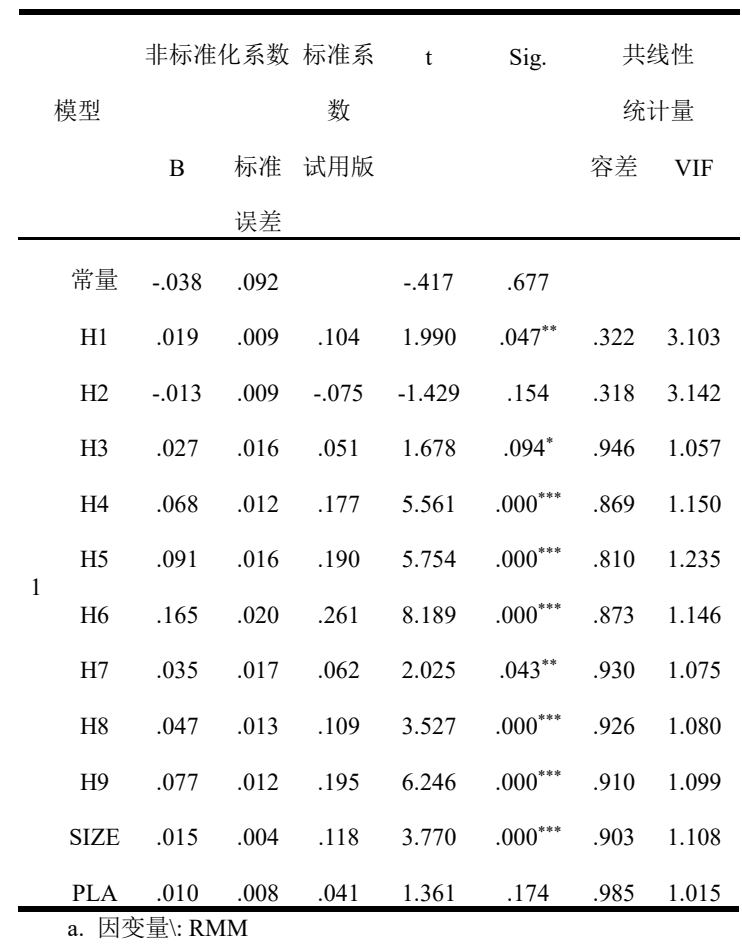

由表 3 我们可以看出, 共线性统计量中容差的值 全部介于 0.3-1 之间, 其中最小的值为 0.318, 所有的 值都大于 $0.1 ; \mathrm{VIF}$ 的值全部介于 1-3.2 之间, 其中最 大的值为 3.142 , 所有的值都小于 10 。这个结果说明 线性回归模型中自变量与自变量之间不存在多重共 线性的情况, 解释变量之间不会由于存在精确相关关 系或高度相关关系而使模型估计失真或难以估计准 确。

线性回归结果方面, 由表 3 中 $\operatorname{Sig}$ 值的表现我们 可以看出: $\mathrm{H} 4 、 \mathrm{H} 5 、 \mathrm{H} 6 、 \mathrm{H} 8 、 \mathrm{H} 9$ 、 SIZE 与 RMM 的 线性回归结果在 $1 \%$ 的水平上显著; $\mathrm{H} 1 、 \mathrm{H} 7$ 与 $\mathrm{RMM}$ 的线性回归结果在 5\%的水平上显著; H3 与 RMM 的
线性回归结果在 $10 \%$ 的水平上显著; H2、PLA 与 RMM 的线性回归结果不显著。

\section{5 结论与建议}

\section{1 研究结论}

公司重大错报风险水平与公司的内部控制存在 重大缺陷或失效、公司存在重大的关联方交易或资金 占用情况、公司存在未决诉讼、仲裁或案件、公司存 在重大的投资、融资或股权转让项目、公司的经营活 动或财务报告受到监管机构的调查或处罚、公司发生 重大收购、重组或其他非经常性事项、公司出现巨额 或连续亏损、公司存在流动负债较多或大额借款未偿 还现象、公司以往存在重大错报风险或本期期末出现 重大会计调整九个指标之间都存在显著的正相关关 系, 说明它们可以作为预测我国上市公司重大错报风 险水平的重要影响因素。

\section{2 应用建议}

上市公司本身可以利用这 9 个重大错报风险影响 因素对公司自身存在的重大错报风险进行合理有效 的规避，降低重大错报风险水平。对于投资者而言， 可以利用这 9 个重大错报风险影响因素对投资目标公 司进行重大错报风险水平的预测, 以此作为投资决策 的参考依据。对于会计师事务所而言, 可以利用这 9 个重大错报风险影响因素对审计目标公司进行重大 错报风险水平的评估, 以此提高审计质量和审计效率。 对于政府监管部门而言, 可以利用这 9 个重大错报风 险影响因素对我国上市公司存在的重大错报风险进 行科学的识别与评估, 以此确定重点监管对象。

\section{REFERENCES}

[1] Wang, X.J. (2016) Risk identification of major misstatement based on the relationship between financial statements check. J. Monthly Journal of Finance and Accounting, 2016(01): 71-73.

[2] Zhang, Y.X., Guo, Y. (2015) Quantitative identification and evaluation of the risk of material misstatement in financial reports: A logistic regression model based on the relative deviation of financial indicators. J. Financial Research, 2015(06): 74-80.

[3] Dhaliwal, D.S., Li, O.S., Tsang, A., et al. (2011) Voluntary non-financial disclosure and the cost of equity capital: The initiation of corporate social responsibility. J. The Accounting Review, 2011, 86(01): 59-100.

[4] Wang, J.L., Li, Y.T., Wu, X. (2016) Corporate social responsibility report and cost of debt capital: Empirical evidence from China's A-share 
market. J. Journal of Shanxi University of Finance and Economics, 2016, 38(07): 113-124.

[5] Song, H.F. (2014) Analysis of the economic consequences of corporate social responsibility information disclosure. J. Accounting News, 2014(18): 96-98. 